\title{
A model of vortex reconnection
}

\author{
By P. G. SAFFMAN \\ Applied Mathematics, California Institute of Technology, Pasadena, CA 91125, USA
}

(Received 19 April 1989)

A model is proposed to describe the breaking and rejoining of vortex lines which occurs when vortex filaments of equal and opposite strengths touch. It is based on the idea that viscosity cancels the vorticity where the filaments first touch. The weakening of the centrifugal force in the vortex core then leads to a local increase in the pressure which accelerates the fluid in the cores in the axial direction and convects the vorticity away from the initial contact and produces an apparent rejoining. Hyperbolic equations to describe the process are formulated and numerical solutions are obtained. Estimates of the timescales are made and compared with the experimental data of Schatzle (1987). Reasonable agreement is found.

\section{Introduction}

The breaking and rejoining of vortex lines is currently a topic of some interest, as an unexpected phenomenon and owing to the belief that it may be a fundamental process in the evolution of three-dimensional vortices and the mechanics of turbulence. Pictures were published by Scorer \& Davenport (1970) of condensation trails exhibiting the phenomenon. The initially straight trails containing the trailing vorticity shed from the airplane's wings develop a sinusoidal oscillation of growing amplitude, known as the Crow (1970) instability. The development to finite amplitude was calculated by Moore (1972). The trails eventually touch and then pinch off, forming a row of vortex rings. Each ring consists of vorticity from both trailing vortices, so that the original vortex lines are broken and rejoin with vorticity from the other trail. Moore's calculations, based on a Biot-Savart cut-off approximation, cease to be valid when the trails touch and the breaking and rejoining is outside the scope of his approach.

Laboratory experiments using colliding vortex rings were carried out by Kambe \& Takao (1971), Oshima \& Asaka $(1975,1977)$, Fohl \& Turner (1975). This work used flow visualization to study how the cores of colliding rings would appear to break and rejoin to fuse into one ring. Under some circumstances, the oscillations of the single ring bring it into a figure-of-eight shape, the cross breaks and rejoins giving fission into two rings, each of which contains half of the original ring. (The Oshima \& Asaka 1975) work demonstrates this elegantly by employing coloured vortex rings.)

Quantitative measurements of colliding vortex rings have recently been carried out by Schatzle (1987). Numerical simulations of the phenomenon, employing finitedifference or spectral methods applied to the Navier-Stokes equations have been carried out by Melander \& Zabusky (1988), Ashurst \& Meiron (1987), Kida \& Takaoka (1988), Meiron et al. (1988) and Kerr \& Hussain (1989). This work has given pictures of the process, but leaves unanswered basic questions about the physics of the breaking and rejoining. Also, because of the difficulty and expense of quantitative experiments and high-resolution numerical simulations of the Navier--Stokes 
equations for large Reynolds number, it is hard to run systematically over a range of parameters, or determine the fundamental scaling of the phenomenon.

The basic questions concern the timescale of the process or processes, the physical mechanisms which drive the reconnection, and the role of viscosity. We present here a simple model which reproduces some of the qualitative features and seems to uncover the basic mechanics. It suggests the existence of two timescales : a first scale in which the cores are forced together and a second scale in which the reconnection takes place. The parameters enter into the equations in a rather complex way and it has not yet proved possible to explore the model analytically. Numerical methods lead to estimates of the timescales, but further progress waits on more quantitative data from numerical simulations.

The model is presented in $\$ 2$. It leads to a set of nonlinear hyperbolic equations. Numerical solutions have been obtained and are described in $\S 3$, together with a comparison with Schatzle's data. Estimates are also made for the dependence of the timescales on the properties of the ring.

\section{The model}

We take the $z$-axis tangential to the axes of the vortex cores when they touch, and the $x$-axis parallel to the line joining the centres. The plane $z=0$ is the plane in which there is closest approach. The motion induced by the rest of the vorticity away from the region of contact (estimatable in principle by use of the Biot-Savart law with cutoff) is assumed to be a locally two-dimensional irrotational strain

$$
u=-\alpha x, \quad v=\alpha y
$$

where

$$
\alpha \approx k \Gamma_{0} / R^{2} \text {. }
$$

Here, $R$ is the radius of the rings or the radius of curvature of the core axes and $k$ depends on the entire geometry. By analogy with the Kelvin formula for the speed of a vortex ring, we can expect that

$$
k \approx \frac{1}{4 \pi} \log \frac{8 R}{a}=O(1) .
$$

(For the Schatzle experiment, $R / a=3.75, k \approx 0.27$.) The cores are translating in the $y$-direction owing to their mutual self-induction. This is clearly seen in Schatzle's photographs. We assume that this translation does not affect significantly the dynamics of the cores. But owing to the induced velocity field (2.1), the cores are squashed together at an exponential rate. We assume that this deforms the cores into elliptical shapes with semi-axes $b(t)$ and $h(t)$ in the $x$ - and $y$-directions. At the same time, viscous diffusion leads to vorticity cancellation, as the vorticity in the cores is of opposite sign. This decreases the rotation and centrifugal forces inside the cores, leading to an increase in pressure relative to the pressure at large distances. Away from the $z=0$ plane of closest approach, the cores will have merged less and the pressure in the vortex cores will remain low. Hence a pressure gradient is set up inside the cores, tending to produce a positive strain $E$ in the $z$-direction. This in turn intensifies the strain bringing the cores together and increases the vorticity diffusion and the pressure. Thus we expect a positive feedback to cancel the vorticity. The process will propagate along the cores, and effectively cut the vortices. The rejoining is not now seen as a separate process, but a consequence of the kinematic theorem that vortex lines cannot end inside a fluid. 
This is the proposed physical mechanism, in which viscous decay generates pressure-driven axial accelerations. The problem is to construct reasonable equations to model it and elucidate the basic physics. We start by assuming that the irrotational straining field, where the vortex cores are merging, has principal rates of strain

$$
e_{x x}=-(\alpha+E), \quad e_{y y}=\alpha, \quad e_{z z}=E,
$$

where $\alpha$ is given by (2.2) and $E$, the axial strain in the cores of the vortices, is to be determined. The model will assume that $E$ is dependent upon $z$ and $t$. Let $\delta(t)$ denote the distance between the core centroids at station $z$, and $b(t)$ and $h(t)$ the minor and major semi-axes of the deformed cores. We now assume the convection equations with viscous diffusion modelled,

$$
\begin{gathered}
\frac{\partial \delta}{\partial t}=-(\alpha+E) \delta+\frac{\nu^{\prime}}{\delta}, \\
\frac{\partial(b h)}{\partial t}=-E b h+\nu^{\prime},
\end{gathered}
$$

where $\nu^{\prime}$ is proportional to the kinematic viscosity $\nu$. Initial conditions at $t=0$ for these variables will be modelled as

$$
\delta=2 a+z^{2} / R, \quad b h=a^{2} .
$$

This assumes that the cores are circular and touching at the start of the process. Validity can only be expected where $z \ll R$.

The vortex cores will have a circulation $\pm \Gamma$ whose decay we model by the equation

$$
\frac{\partial \Gamma}{\partial t}=-\frac{\nu^{\prime} \Gamma}{\delta^{2}} .
$$

The accuracy of (2.8), and (2.11) and (2.12) below for the pressure in the cores, can be tested by studying solutions of the Navier-Stokes equations for stretched vortices in a uniform strain; prototypes are the Burgers-Townsend exact solutions. A calculation of this type has recently been carried out by Buntine \& Pullin (1989). Our model predicts circulation decay $\Gamma \sim \mathrm{e}^{-(\alpha+E) t}$ as $t \rightarrow \infty$ for the case $E=$ const. and $\partial / \partial z=0$, which agrees well with qualitative decay of circulation seen in the Buntine \& Pullin results. (Viscosity does not explicitly appear in this expression since $\delta \sim \nu^{\frac{1}{2}}$.) Pullin has kindly pointed out that quantitative agreement is also found for the behaviour of $\Gamma$ and $\delta$ if we take $\nu^{\prime}=2 \pi \nu$, which we shall henceforth do. There may, however, be disagreements in the shape of the vortex cores. by

We now need an equation for $E(z, t)$. the $z$-momentum equation is approximated

$$
\frac{\partial w}{\partial t}=-\frac{\partial p}{\partial z}-w \frac{\partial w}{\partial z}
$$

where $w$ and $p$ are respectively an average axial velocity and pressure over the cores. Then we take

$$
E=\partial w / \partial z
$$

The system of equations is now closed by an expression for $p$, which we assume depends upon the core deformation. The magnitude of the pressure drop at the centre of an elliptical vortex is, on dimensional grounds,

$$
P=\Gamma^{2} f(\theta) / 2 \pi^{2} b h,
$$

where $\theta=h / b$ is the axis ratio. The Kirchhoff solution for a rotating elliptical vortex 
and the generalization by Moore \& Saffman (1971) to an elliptical vortex in a straining field suggest the approximation $f=\theta /\left(1+\theta^{2}\right)$ for $\theta>1$ (see the Appendix). Further, the Moore \& Saffman expression for $\theta$ in terms of the ratio of external strains to vorticity suggests the approximation

$$
\theta=1+4 r, \text { where } r=\pi \alpha b h / \Gamma+b h / \delta^{2} .
$$

The first term in the expression for $r$ describes the effect of external strain and the second term is an estimate of the deformation caused by the extra strain produced by the other filament.

We take $p=-P$, and the set of equations is now complete. There are four equations, (2.5), (2.6), (2.8) and (2.9), for the four variables, $\delta, b h=A, \Gamma$, and $w$. The initial conditions (2.7) together with

$$
\Gamma=\Gamma_{0}, \quad w=0 \quad \text { when } t=0
$$

are sufficient in principle to determine a solution.

Typical values of the parameters based on Schatzle's experiments are $\Gamma=$ $16 \mathrm{~cm}^{2} / \mathrm{s}, \quad R=1.5 \mathrm{~cm}, a=4 \mathrm{~mm}, v=0.01 \mathrm{~cm}^{2} / \mathrm{s}$. The formulation suggests (as described in $\S 3$ ) that the equations be analysed in terms of two small parameters

$$
\epsilon=a / R, \quad \mu=\pi v^{\prime} /\left(\Gamma_{0} \epsilon^{\frac{1}{2}}\right) .
$$

The first is a geometric parameter of the vortex rings, and the second is essentially an inverse Reynolds number. For Schatzle's data, $\epsilon \approx 0.27, \mu \approx 0.025$.

In an attempt to construct a relatively simple model of a rather complicated phenomenon, there are always going to be questionable choices. For example, a referee has suggested that the operator $\partial / \partial t$ should be replaced by $\partial / \partial t+w \partial / \partial z$ in $(2.6)$ and (2.8). The present choice was made on the grounds that area and circulation are global properties of the core and are convected by the flow external to the core, but this point and others like it are certainly arguable. Test of the model have indicated that it is relatively robust, and its predictions are not particularly sensitive to changes of this nature.

\section{Scaling of the model}

We make the variables dimensionless, retaining the same symbols, and putting $A=b h$. For $\delta, b, h$, we scale on $a$, the initial core radius. For $z$, we scale on $(a R)^{\frac{1}{2}}$. We scale $\Gamma$ on $\Gamma_{0}, t$ on $\left(\pi a^{2} / \Gamma_{0}\right)(R / a)^{\frac{1}{2}}$, and $w$ on $\Gamma_{0} / \pi a$. These choices were found by trial and error to give suitable balances of the terms. The dimensionless evolution equations then become for the variables $\delta(z, t), A(z, t), \Gamma(z, t), w(z, t)$ :

$$
\begin{gathered}
\frac{\partial \delta}{\partial t}=-\left(\epsilon^{\frac{3}{2}}+\frac{\partial w}{\partial z}\right) \delta+\frac{\mu}{\delta} \\
\frac{\partial A}{\partial t}=-\frac{\partial w}{\partial z} A+\mu \\
\frac{\partial \Gamma}{\partial t}=-\frac{\mu \Gamma}{\delta^{2}} \\
\frac{\partial w}{\partial t}=-w \frac{\partial w}{\partial z}+\frac{\partial}{\partial z}\left(\frac{f \Gamma^{2}}{2 A}\right)
\end{gathered}
$$


with the relations

$$
f=\theta /\left(1+\theta^{2}\right), \quad \theta=1+4 r, \quad r=A / \delta^{2}+\epsilon^{2} A / \Gamma,
$$

and initial conditions at $t=0$

$$
\delta=2+z^{2}, \quad A=1, \quad \Gamma=1, \quad w=0 .
$$

The small parameters $\epsilon$ and $\mu$ are given in (2.14). In these equations, it was assumed for definiteness that $k \pi=1$ (see (2.3)) as the value appropriate for colliding rings. For other geometries, e.g. the touching of trailing vortices due to the Crow instability, other values of $k$ may be appropriate. Tests showed no significant dependence on the value of $k$.

In terms of the dimensionless variables, the dimensional axial strain, vorticity, axial distance, and time are

$$
E^{*}=\epsilon^{-\frac{3}{2}} \alpha \frac{\partial w}{\partial z}, \quad \omega^{*}=\frac{\epsilon^{-2} \alpha \Gamma}{A}, \quad z^{*}=\epsilon^{-\frac{1}{2}} a z, \quad t^{*}=\frac{\epsilon^{\frac{3}{2}} t}{\alpha},
$$

where henceforth an asterisk denotes a dimensional variable.

The system of equations is hyperbolic, and was solved numerically using centred differences in $z$ and simple Euler in $t$. We employed a range of $z$ from 0 to a value $z_{\max }$ so that the solution of interest was independent of $z_{\max }$. A value of 20 seemed adequate. It was found by testing that 400 grid points in the $z$-direction were sufficient to give results independent of the $z$-mesh. It is sufficient to consider only $z>0$ because of the symmetry. The time step $\delta t$ was chosen to satisfy the CFL condition $\delta t<\delta z / c_{\max }$, where $c_{\max }$ is the largest wave speed. A value of $10^{-4}$ for $\delta t$ was found to give results unaffected by further reduction. More sophisticated integration schemes could be employed, but did not appear to be called for in view of the simplicity of the model. One of the principal questions is, obviously, to determine if $\Gamma$ falls to zero in a finite time, and if it does to find the trajectory in the $(z, t)$-plane on which $\Gamma=0$. It is clear from (3.3) that $\Gamma$ cannot fall to zero unless $\delta$ goes to zero simultaneously, and $E$ goes to infinity and $A$ to zero. There may be a solution with these properties, but we shall assume that it is not an 'attractor', and that breaking of the vortex lines can be recognized by $\Gamma$ dropping below $5 \%$ say.

In figure 1 , we show a plot of $z_{\mathrm{c}}$, and of $\delta, \Gamma, A$, and $E$ on the centreplane $z=0$, as functions of time, where $z_{\mathrm{c}}$ is the largest value of $z$ for which $\Gamma<0.05$ and is taken to mark the boundary of the rejoining region. The values of $\epsilon$ and $\mu$ are those for Schatzle's data. The timescale $T_{1}$ for $\Gamma$ to become negligibly small is about 12 . The connection then propagates along the cores and the time $T_{2}$ for $z_{\mathrm{c}}$ to go from 0 to 1 is about 7. As the circulation in the core decays, the axis ratio given by (3.5) increases and the core flattens, in qualitative agreement with the observations.

In dimensional units, the time to connection is

$$
T_{1}^{*}=\frac{\pi a^{2}}{\Gamma_{0}}\left(\frac{R}{a}\right)^{\frac{1}{2}} T_{1}
$$

For values of the parameters corresponding to Schatzle's data, $T_{1}^{*}$ is about $0.75 \mathrm{~s}$, which agrees well with the times for the vorticity in the cores to mingle shown in figure 6.11 of Schatzle's thesis (pp. 148-151). It should be noted, however, that there is a significant discrepancy between the magnitudes of the maximum vorticity predicted by the model during the core merging process. According to the data shown in figure 1 , the vorticity in the cores will roughly double in magnitude, while 


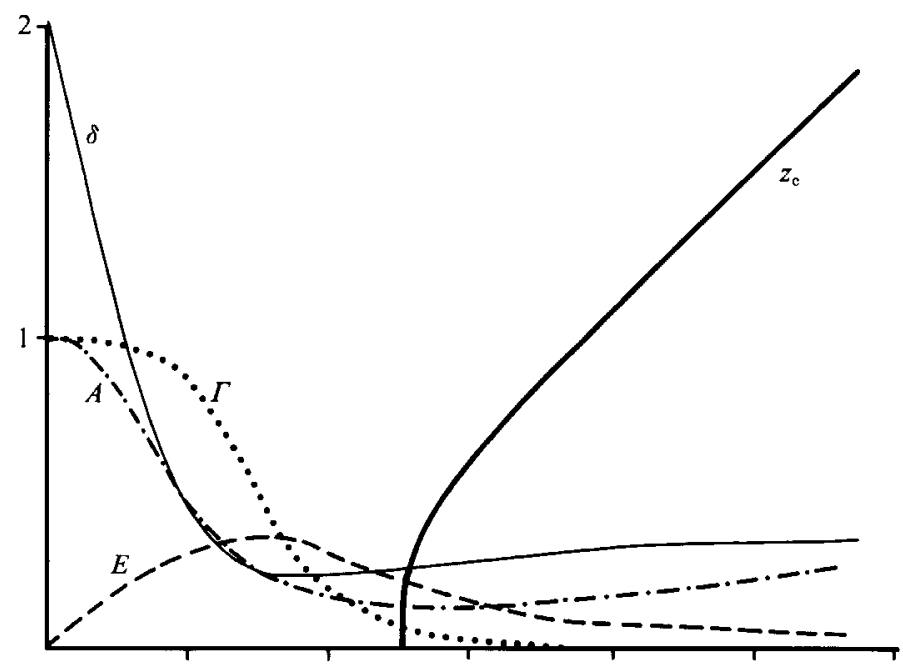

Figure 1. Values of $\delta, \Gamma, A, E v s . t$ on the centreplane $z=0:-, \delta ; \cdots \cdots, \Gamma ; \cdot \cdot \cdot-\cdot-\cdot, A$; $E ;-, z_{\mathrm{c}}$, the locus of rejoining station. $\epsilon=0.27, \mu=0.025$.

Schatzle's data show hardly any increase. On the other hand, the numerical simulations (e.g. Kerr \& Hussain 1989) do show increases in the' magnitude of the vorticity of this order. This discrepancy between the experimental data and the model and simulations is not understood.

The dimensional rejoining time

$$
T_{2}^{*}=\frac{\pi a^{2}}{\Gamma_{0}}\left(\frac{R}{a}\right)^{\frac{1}{2}} T_{2} .
$$

Our predicted time for rejoining for Schatzle's parameters is approximately $0.4 \mathrm{~s}$. This appears to be twice as long as the times for breaking in the photographs shown in Schatzle's figure 1.2, frames 4, 5 and 6.

Comparison of the calculated and measured rates of strain is reasonable. The computations show that $E$ reaches a maximum of about 0.35 shortly before the circulation becomes negligibly small. Then

$$
E^{*} / \alpha=E \epsilon^{-\frac{3}{2}} \approx 2.5 .
$$

Then the dimensional values of the principal rates of strain (see (2.4)) are $-3.5 \alpha, \alpha$, 2.5 $\alpha$. Taking $\alpha=2 \mathrm{~s}^{-1}$ from (2.2), this gives the values of about $-7,2,5$. Schatzle's data shown in his figures 6.6,6.9 and 6.10 suggest values of about $-6,2,6$. (Note that our coordinate system differs from that of Schatzle; our $\partial w / \partial z$ is his $\partial v / \partial y$.

The main discrepancies between the model and the experiments are the absence in the experiment of vorticity amplification and the timescale $T_{2}$ for the rejoining, which seems to be too large by a factor of two. We do not know if these are due to a significant flaw in the model or difficulties in the measurement and interpretation of the data. It should be noted that trials indicated that the values of $T_{2}$ are insensitive to the values of the various coefficients of proportionality which have been put equal to unity in the model equations. With regard to possible uncertainties in the interpretation of data, the distribution of dye in the water may not describe closely the distribution of vorticity, and absence of dye may not imply absence of vorticity. 
The equations have been solved for various values of $\epsilon$ and $\mu$ in the range 0.27 to 0.1 and 0.001 to 0.025 , respectively. The results suggest that $T_{1} \sim 5 \epsilon^{-\frac{1}{4}}[\ln (1 / \mu)]^{\frac{1}{2}}$ and that $T_{2} \sim 2 / \epsilon$, but these conclusions need to be confirmed. The dimensional times then behave like

$$
T_{1}^{*} \sim \frac{5 \pi R^{2}}{\Gamma_{0}} \epsilon^{\frac{5}{4}}\left(\ln \frac{1}{\mu}\right)^{\frac{1}{2}}, \quad T_{2}^{*} \sim \frac{2 \pi R^{2}}{\Gamma_{0}} \epsilon^{\frac{1}{2}}
$$

It is hoped that further simulations may be available before long to allow testing of the model.

This paper is dedicated with respect and appreciation to my thesis advisor, Professor G. K. Batchelor, to commemorate his extensive and valuable contributions to Fluid Dynamics over many years.

I wish to thank Professor D. Meiron, Professor D. W. Moore, and especially Dr D. I. Pullin for the benefit of discussions on the model and for valuable suggestions. The work was supported by the Department of Energy, Applied Mathematical Sciences (DE-AS03-76ER72012 KC-07-01-01).

\section{Appendix. Pressure in a strained vortex}

Consider a two-dimensional patch of constant vorticity $\omega$ in an inviscid fluid. The stream function can be written $\psi=\Psi+\psi^{\prime}$, where $\nabla^{2} \Psi=-\omega$, and $\psi^{\prime}$ is harmonic. If $\phi^{\prime}$ denotes the harmonic conjugate of $\psi^{\prime}$, it is well known that the pressure satisfies a generalization of Bernoulli's equation,

$$
\partial \phi^{\prime} / \partial t+\frac{1}{2} q^{2}+\omega \psi+p=0
$$

Consider an elliptical vortex with semi-axes $h$ and $b(h>b)$ of uniform vorticity $\omega_{0}$ at rest in a uniform straining field $u=-\alpha y, v=-\alpha x$. The stream function $\psi$ inside the vortex is

$$
\psi=-\frac{\omega_{0} h^{2} b^{2}}{2\left(h^{2}+b^{2}\right)}\left(\frac{x^{2}}{h^{2}}+\frac{y^{2}}{b^{2}}-1\right) .
$$

In the case of steady flow, we have $p+\frac{1}{2} q^{2}=H$ outside the vortex, and $p+\frac{1}{2} q^{2}+$ $\omega \psi=H$ inside the vortex. Hence at the centre of the vortex, the excess pressure $-P$ is given by

$$
P=\omega \psi=\frac{\omega_{0}^{2} h^{2} b^{2}}{2\left(h^{2}+b^{2}\right)} .
$$

This is the formula used in (2.11).

\section{REFERENCES}

Ashurst, W. T. \& Metron, D. I. 1987 Numerical study of vortex reconnection. Phys. Rev. Lett. $58,1632-1635$.

Buntine, J. D. \& Pullin, D. I. 1989 Merger and cancellation of strained vortices. J. Fluid Mech. 205, 263-295.

Crow, S. C. 1970 Stability theory for a pair of trailing vortices. AIAAJ. 8, 2172-2179.

Fohl. T. \& Turner, J. S. 1975 Colliding vortex rings. Phys. Fluids 18, 433-436.

KaMBe, T. \& TakaO, T. 1971 Motion of distorted vortex rings. J. Phys. Soc. Japan 31, 591-599.

KERR, R. M. \& Hussain, F. 1989 Simulation of vortex reconnection. Physica $D$ (to appear).

KIDA, S. \& TAKAOKA, M. 1988 Reconnection of vortex tubes. Fluid Dyn. Res. 3, 257-261. 
Meiron, D. I., Sheltey, M. J., Ashurst, W. T. \& Orszag, S. A. 1988 Numerical studies of vortex reconnection. Proc. SIAM Workshop on Mathematical Aspects of Vortex Dynamics, Leesburg VA. SIAM Proceedings.

Melander, M. V. \& ZABusky, N. J. 1988 Interaction and 'apparent' reconnection of 3D vortex tubes via direct numerical simulations. Fluid Dyn. Res. 3, 247-250.

Moore, D. W. 1972 Finite amplitude waves on aircraft trailing vortices. Aero. Q. 23, 307-314.

Moore, D. W. \& Saffman, P. G. 1971 Structure of a line vortex in an imposed strain. In Aircraft Wake Turbulence and its Detection (ed. J. H. Olsen, A. Goldburg \& M. Rogers), pp. 339-354. Plenum.

Oshima, Y. \& Asak A, S. 1975 Interaction of two vortex rings moving side by side. Nat. Sci. Rep. Ochanomizu Univ. 26, 31-37.

Oshima, Y. \& AsakA, S. 1977 Interaction of two vortex rings along parallel axes in air. J. Phys. Soc. Japan 42, 708-713.

SChAtZLE, P. R. 1987 An experimental study of fusion of vortex rings. Ph.D. thesis, California Institute of Technology.

Scorer, R. S. \& Davenport, L. J. 1970 Contrails and aircraft downwash. J. Fluid Mech. 43, 451-464. 